\title{
Editorial
}

\section{Networked Systems with Complexities and Their Applications to Engineering}

\author{
Dong Yue, ${ }^{1}$ Engang Tian, ${ }^{2}$ and Yang Tang ${ }^{3}$ \\ ${ }^{1}$ College of Automation Engineering, Nanjing University of Posts and Telecommunications, Nanjing 210046, China \\ ${ }^{2}$ School of Electrical and Automation Engineering, Nanjing Normal University, Nanjing 210042, China \\ ${ }^{3}$ Institute of Physics, Humboldt University Berlin, 12489 Berlin, Germany
}

Correspondence should be addressed to Dong Yue; medongy@njnu.edu.cn

Received 11 June 2013; Accepted 11 June 2013

Copyright (C) 2013 Dong Yue et al. This is an open access article distributed under the Creative Commons Attribution License, which permits unrestricted use, distribution, and reproduction in any medium, provided the original work is properly cited.

The aim of this special issue is to highlight the most significant recent developments on the topics of networked systems, especially networked control systems, multiagent systems, and cyber-physical systems, their modeling, identification, stability analysis, and estimation facing with various problems, such as random communication delay, packet loss, data quantization, saturation, communication constraints, random faults, and switched systems. The topics to be covered include the following: networked control systems (mathematical modeling, event-triggered control, stochastic control, fault detection and diagnosis, scheduling algorithm, control and communication codesign, quantized control, nanosensors, multichannel transmission, etc.); multiagent systems (consensus, cooperation, filtering, flocking, synchronization, pinning control, formation control, evolutionary computation, particle swarm optimization, etc.); cyber-physical systems (wireless sensor network, internet of things, smart grid, intelligent transportation system, system of system, etc.).

Dong Yue

Engang Tian Yang Tang 


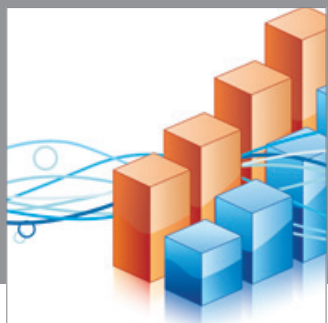

Advances in

Operations Research

mansans

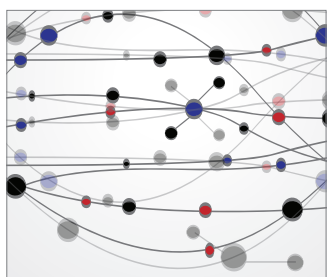

The Scientific World Journal
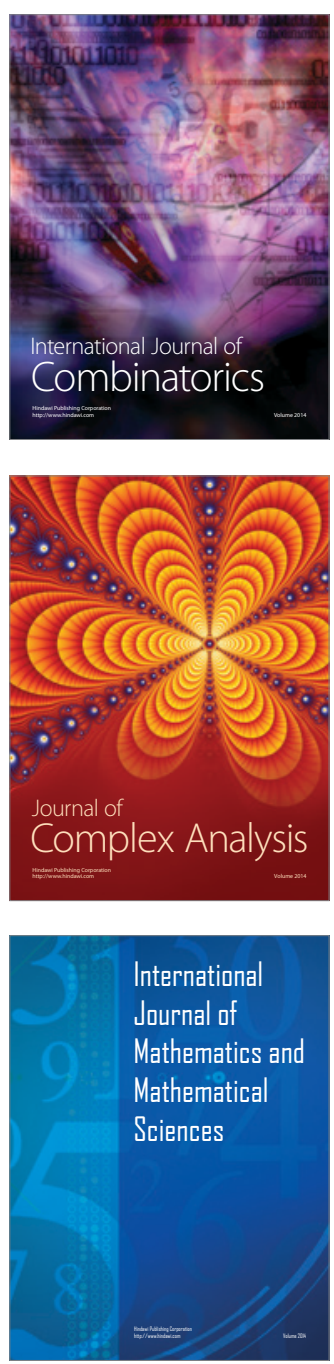
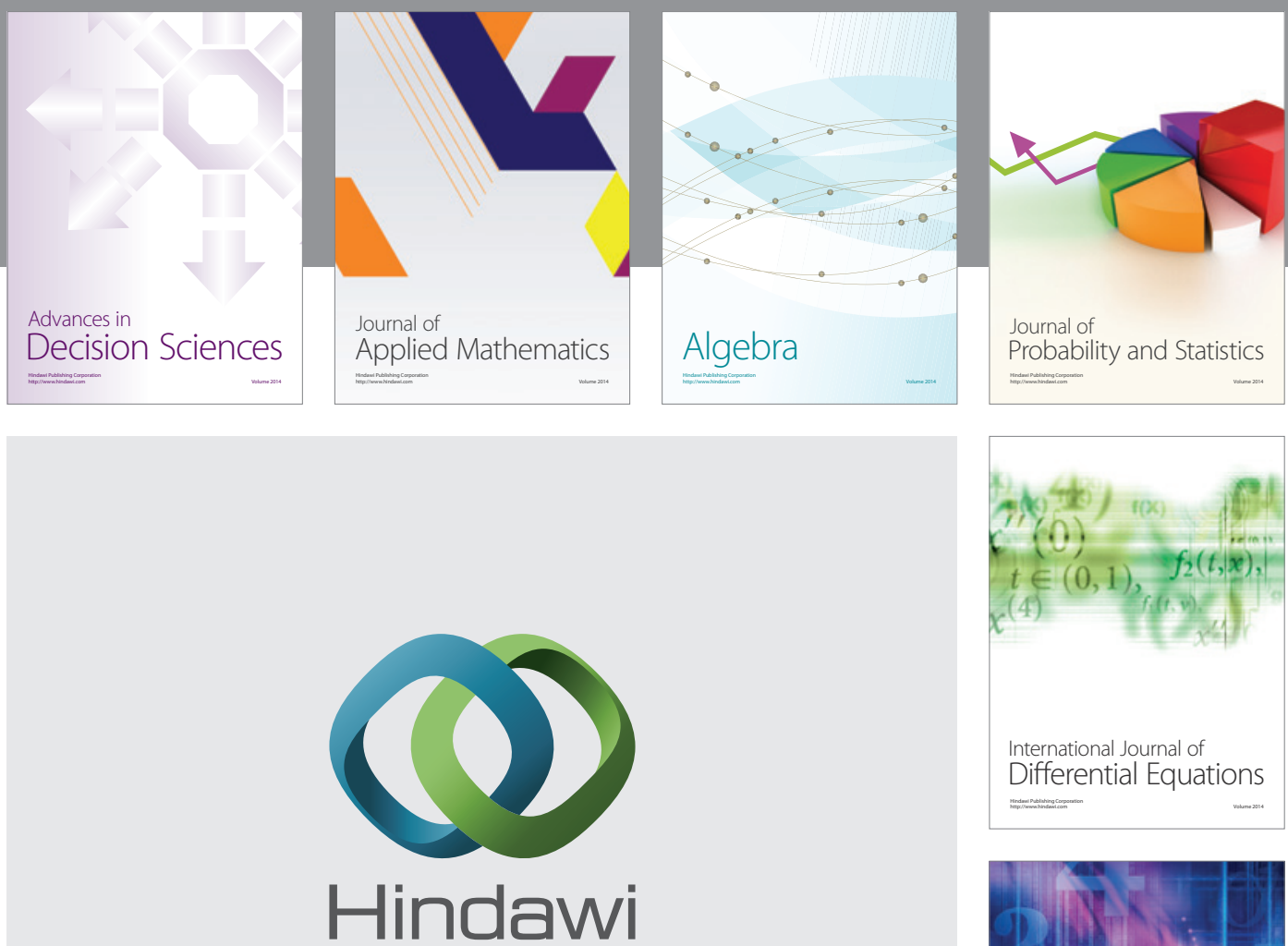

Submit your manuscripts at http://www.hindawi.com
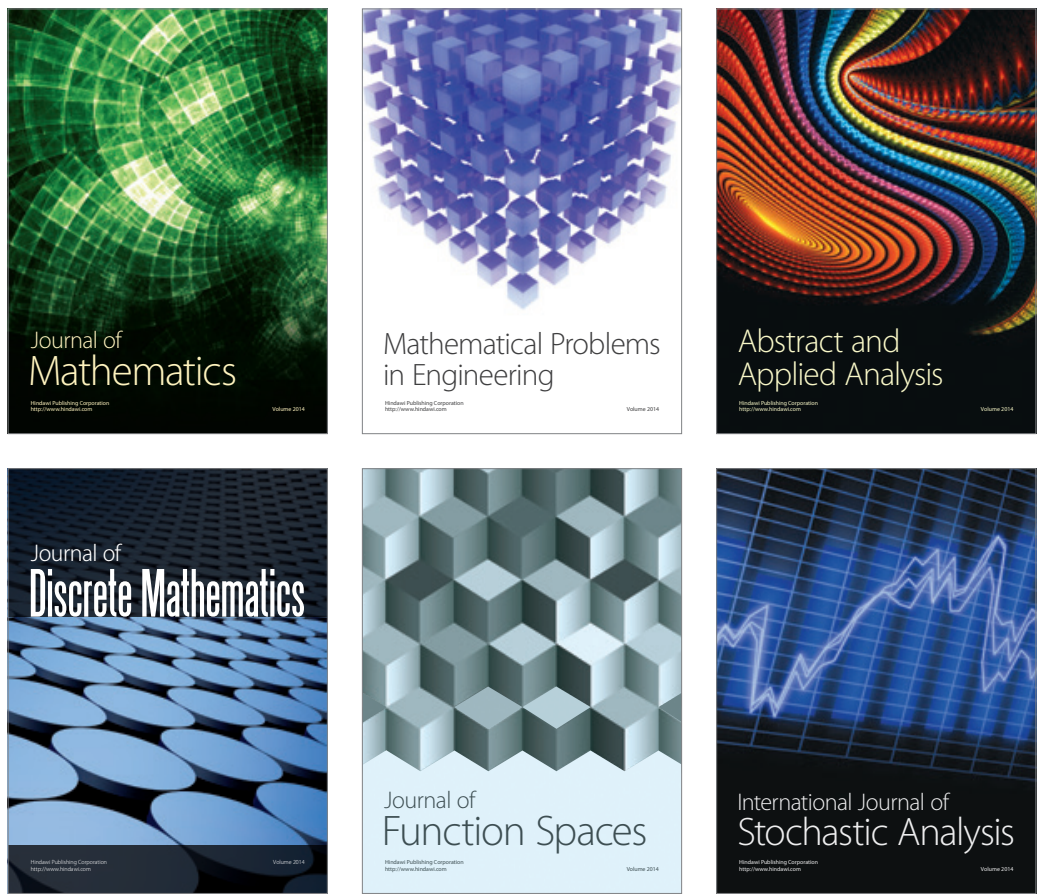

Journal of

Function Spaces

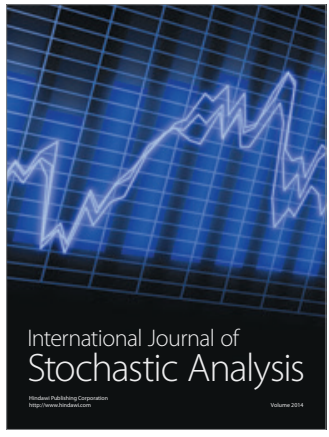

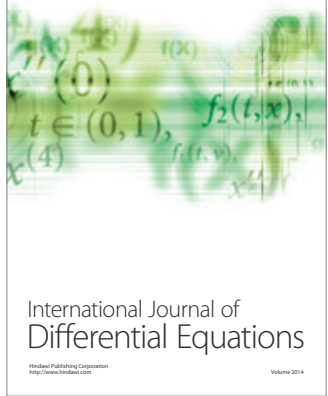
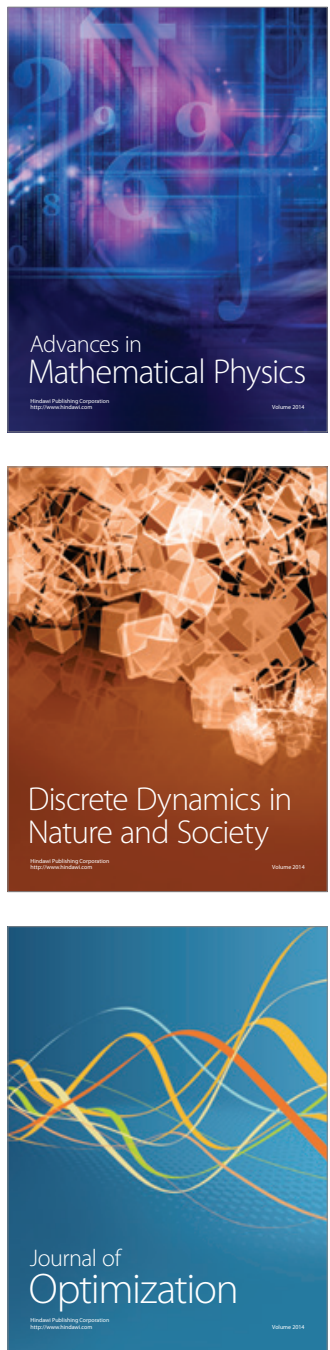